\title{
Modified screen-printed Electrode as Electrochemical Detector for Noscapine
}

\author{
Mohammad Reza Aflatoonian ${ }^{1,2}$, Peyman Mohammadzadeh Jahani ${ }^{* 3}$, Behnaz Aflatoonian ${ }^{4}$, Maedeh \\ Jafari $^{5}$, Kaiqiang Zhang ${ }^{* 6}$, Quyet Van Le ${ }^{* 7}$, Joo Hwan Cha ${ }^{8}$, Mohammadreza Shokouhimehr ${ }^{9}$ and \\ Wanxi Peng ${ }^{* 10,11}$
}

${ }^{1}$ Research Center for Tropical and Infectious Diseases, Kerman University of Medical Sciences,

Kerman, Iran

${ }^{2}$ Leishmaniasis Research Center, Kerman University of Medical Sciences, Kerman, Iran

${ }^{3}$ School of Medicine, Bam University of Medical Sciences, Bam, Iran

${ }^{4}$ Neuroscience Research Center, Kerman University of Medical Sciences, Kerman, Iran

${ }^{5}$ Department of Pediatrics, School of Medicine, Kerman University of Medical Sciences, Kerman, Iran ${ }^{6}$ Jiangsu Key Laboratory of Advanced Organic Materials, Key Laboratory of Mesoscopic Chemistry of MOE, School of Chemistry and Chemical Engineering, Nanjing University, Nanjing, Jiangsu 210023, China

${ }^{7}$ Institute of Research and Development, Duy Tan University, Da Nang 550000, Vietnam

${ }^{8}$ Innovative Enterprise Cooperation Center, Korea Institute of Science \& Technology, Hwarangro 14gil, Seongbuk-gu, Seoul, Korea

${ }^{9}$ Department of Materials Science and Engineering, Research Institute of Advanced Materials, Seoul National University, Seoul 08826, Republic of Korea

${ }^{10}$ College of Forestry, Henan Agricultural University, Zhengzhou 450002, China

${ }^{11}$ School of Automotive Engineering, Huanghe Jiaotong University, Jiaozuo 454950, China

*E-mail: mjpeyman@yahoo.com, kaiqiangzhang126@126.com, Levanquyet@dtu.edu.vn, pengwanxi@163.com

doi: $10.20964 / 2020.09 .58$

Received: 17 April 2020 / Accepted: 6 July 2020 / Published: 10 August 2020

In the present study, a $\mathrm{La}^{3+}$ doped $\mathrm{Co}_{3} \mathrm{O}_{4}$ nanoflowers modified graphite screen-printed electrode $\left(\mathrm{La}^{3+}\right.$ doped $\mathrm{Co}_{3} \mathrm{O}_{4}$ nanoflower/SPE) were prepared and used for voltammetric determination of noscapine. Presence nanoflowers lead to a substantial improvement in current in compare to an unmodified electrode. The diagnostic methods used for the purposes of the study include chronoamperometry, differential pulse voltammetry (DPV) and cyclic voltammetry (CV) approaches. The modified SPE was used to determine noscapine in the range of 0.3 to $600.0 \mu \mathrm{M}$ (LOD=0.1 $\mu \mathrm{M}$ ).

Keywords: Screen printed electrode, Noscapine, Modified electrode, $\mathrm{La}^{3+} / \mathrm{Co}_{3} \mathrm{O}_{4}$ nanoflower, Voltammetry, 


\section{FULL TEXT}

(C) 2020 The Authors.Published by ESG (www.electrochemsci.org). This article is an open access article distributed under the terms and conditions of the Creative Commons Attribution license (http://creativecommons.org/licenses/by/4.0/). 